\title{
Analysis of Ocular Blood Flow Autoregulation in Human by Using an ARX Model
}

\author{
Jintao $\mathrm{Yu}^{1}$, Zhiping Yang ${ }^{1}$, Lin Wang ${ }^{2}$ and Fang Feng ${ }^{1}$ \\ ${ }^{1}$ School of Computer and Information Engineering, Harbin University of \\ Commerce, Harbin, China 150028 \\ 2. Discoveries in Sight Research Laboratories, Devers Eye Institute, Legacy \\ Research Institute, Portland, Oregon \\ hityjt@sina.com
}

\begin{abstract}
The aim of this study was to establish an ARX model to describe the relationship between the arterial blood pressure $(A B P)$ and the ocular blood flow $(B F)$ in the optic never head $(\mathrm{ONH})$ under the experimental condition of $A B P$ suddenly changes. The sudden changes in ABP was induced by the method of the thigh cuff. The parameters of this model can be estimated by using system identification. In this paper, recursive least squares algorithm was been adapted.

The theoretical and actual data fitted welland suggest that this model can effectively describe both the linear and nonlinear featire in dynamic and static autoregulation in the $O N H$. It shows that the BF autoregulation wully functions when the $A B P$ was $>80 \mathrm{mmHg}$ and works incompetely when $A B P$ yous 80 mmHg. This model may be used to help investigating the features of autoregulation in the $\mathrm{ONH}$ under different experimental conditions.
\end{abstract}

Keywords: system identification, autoregulation, ARX, the thigh cuff

\section{Introduction}

Glaucoma represents the second cause of blindness in the world.The estimated number of patients suffermg from this disease is about 68 million, of whom 6,7 million are blind[1-2]. Im order to cure glaucoma, we need to understand the ocular autoregulative mechanisms firstly.

Abnormalities in blood flow regulation in ONH have been implicated in a variety of eye diseases induding glaucoma, diabetes and age-related muscular degeneration [3-10]. So assessment of autoregulation (AR) in ocular tissues is important to understand the mechanisms of diseases and be beneficial to determine the treatment method.

Ocular autoregulation is the physiological system controlled by myogenic, metabolic, neurgenic and other unknown mechanisms, which maintains ocular blood flow at an approximately constant level despite limited changes in the arterial blood pressure (ABP) and intraocular pressure (IOP)[11].

Changes in ABP that may be used include spontaneous and manipulated in a step-wise or sinusoidal manner. In human, a sudden drop in ABP can be induced by releasing a previously inflated thigh cuff. This method has been used to observe dynamic autoregulation in the cerebral circulation, and has been adopted in previous studies to induce acute changes in ocular perfusion pressure to enable dynamic autoregulation in $\mathrm{ONH}$ to be assessed.

When the ABP step challenge is applied rapidly, the $\mathrm{ONH}$ autoregulation can be classified into two phases of hemodynamic responses according to the time taken to accomplish blood flow adjustment: dynamic autoregulation (dAR) and static 
autoregulation (sAR).The dAR phase which is an initial dynamic phase lasts on average 10-20 sec when the vascular smooth muscles react to adjust the vascular resistance in an attempt to recover the state of $\mathrm{BF}$ to its original level or a new level[12,13]. The sAR phase is reached later when the dynamic BF changes have equilibrated to a steady state level. The sAR phase may involve many factors to adjust the blood flow, since there is sufficient time to involve metabolic, myogenic and neurogenic factors to adjust the blood flow.

A series of $\mathrm{BF}$ changes measured during the $\mathrm{AR}$ in response to varying levels of $\mathrm{ABP}$ constitute a classic sAR curve as demonstrated in Figure 1.To data, most previous studies to assess the autoregulation capacity in ocular tissues has been limited to sAR, the involvement of dAR is not yet fully understood. Analysis of dAR can provide insights into the time course of the autoregulatory response.The inherent complexities of ocular tissue encourage the use of mathematical modeling to describe the autoregulation capacity.

Utilizing the mechanistic features of autoregulation, discrepancies between theoretical systems behaviors and actual behaviors measured in normal subjects can poin to the hitherto unknown components that are missing, thereby assisting in deyeloping a more comprehensive picture of this biological process.

However, studies that investigated autoregulatory behavior in ONH are parse. We can learn from the experience about the studies of modeling of cerebral blood flow autoregulation.

Some ARX model has been used to simulate the autoregulatory response of cerebral blood flow to a sudden drop in ABP produced by deflation of thigh cuff.

From some researches from cerebral blood flow autoregulation system ,it can be seen that a higher order of ARX model may be more appropriate to represent the BF autoregulation system since it is capable of providing more flexibility to describe both linear and nonlinear features of th $\varnothing \mathrm{BF}$ autoregulation.

In this paper, we assume an $\mathrm{ARX}$ model which is established to describe the ABP-BF relationship incorporating both $\mathrm{AAR}$ and $\$ \mathrm{AR}$. The order of this model was determined by a theoretical three-order parametric which was .assumed by analysing the physiological ocular blood flow, autoregulation system combined with some conclusions by other researchers.

The resultant ARX model may provide clinical tools for diagnosis, monitoring and prognosis of patients with ege diseases.

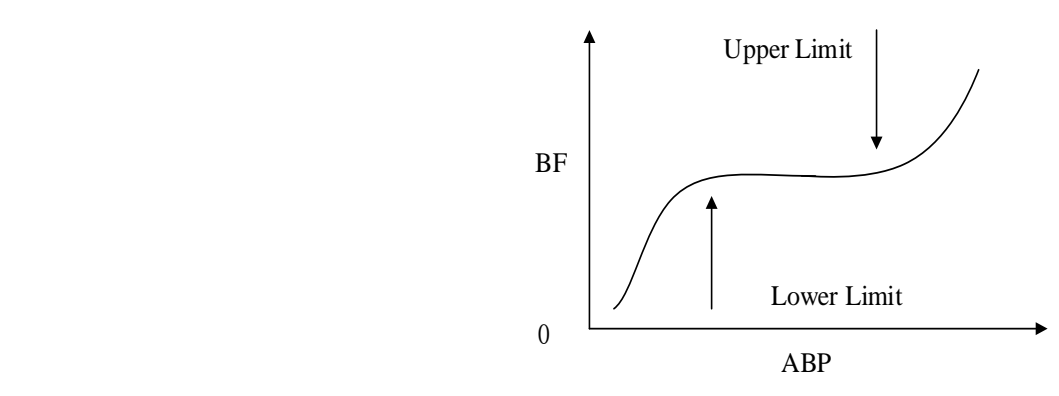

Figure 1. Static Autoregulation Curve. BF Is Kept Constant in Autoregulatory Range. When ABP Fluctuations Exceed this Range, I.E.Higher than the Upper Limit or Lower Than The Lower Limit, BF Will Passively Increase Or Decrease 


\section{Materials and Methods}

\subsection{Subjects and Preparation}

14 subjects without eye and cardiovascular diseases, ranging from 23 to 54(41.4 \pm 11.2 , mean $\pm \mathrm{SD}$ ) years old and height from 152 to $185(170.2 \pm 8.1) \mathrm{cm}$ were participated in this study. For each subject, the experiment was repeated three times, and data were consecutively recorded to maintain a relative steady state of $\mathrm{ONH}$ autoregulation.

The BF of the ONH was measured by a laser speckle flow graph(LSFG,NAVI LSFG system, Softcare,Japan) (Figure 2).This device consists of a fundus camera equipped with a diode laser and a halogen lamp.The halogen is used to define the area to be examined; the laser $(\lambda=830 \mathrm{~nm}$, maximum output power, $1.2 \mathrm{~mW})$ is switched on at the time of measurement.The measured fundus area is approximately $3.8 \times 3 \mathrm{~mm}$ (width $\times$ height), with an eatimated depth of tissue penetration of 0.5 to $1 \mathrm{~mm}$, bases on estimations in buman eyes. ABP was recorded at $200 \mathrm{~Hz}$ sampling rate with a noninvasive blood press monitor(Finometer Pro@, Holland) (Figure 3) by placing a finger cuff around one of the fingers. At the same time, $\mathrm{BF}$ was recorded at $30 \mathrm{~Hz}$ sampling rate.

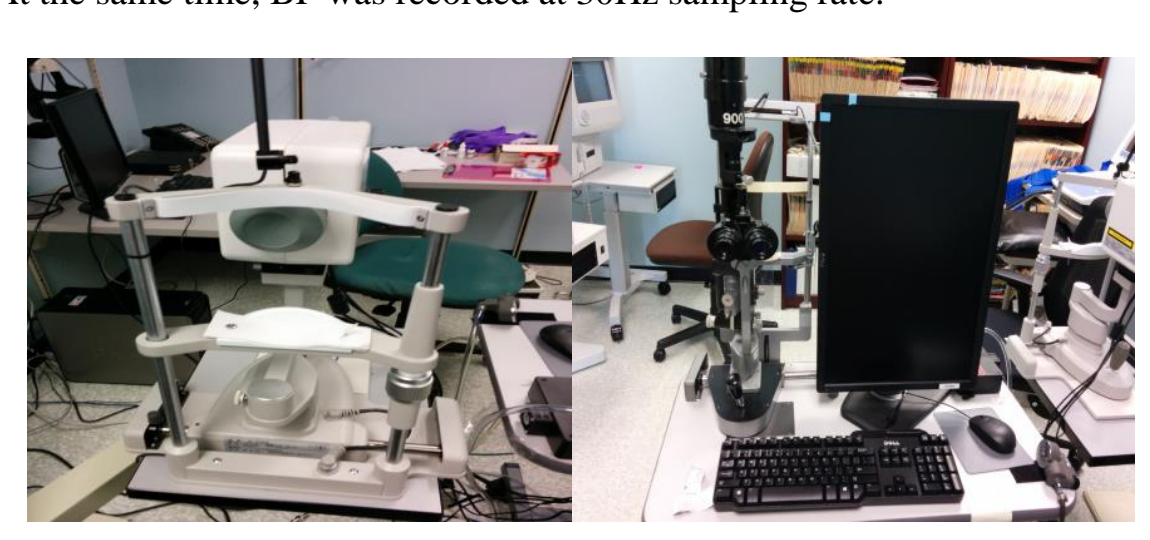

Figure 2. LSFG

Figure 3. Finometer Pro

\subsection{Data Preparation}

The beat-to-beat pulsatities of measured $\mathrm{ABP}$ and $\mathrm{BF}$ were removed using a seventh-order ellipptic low-pass filter. The pass band frequency was $0.45 \mathrm{~Hz}$ and the stop-pass frequency $0.55 \mathrm{~Hz}$, with $40 \mathrm{~dB}$ attenuation in the stop band. The ripple amplitude of the pass band was $<0.01 \mathrm{~dB}$. The filted data were down-sampled at $1 \mathrm{~Hz}$ to increase numerical robustness in parameter estimation. The data after filtering are shown in Figure 4. 

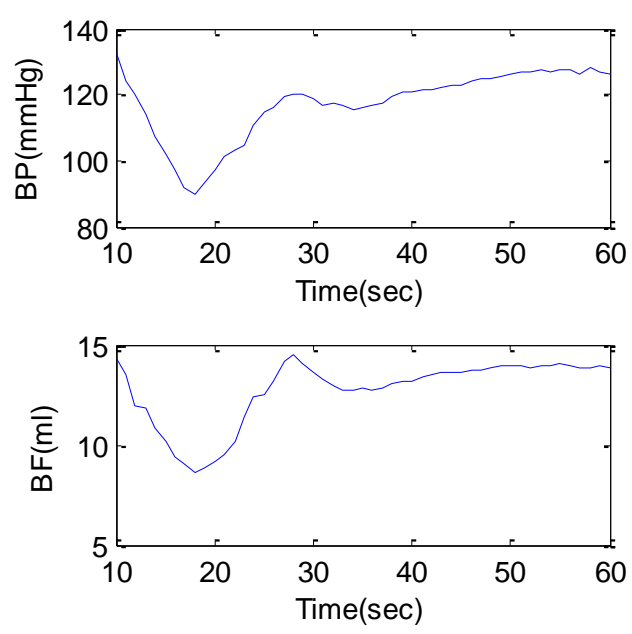

Figure 4. The Recording of BP and BF after Data Preparation BP Was Acutely Decreased Starting At 10th Second and then Recoveryed to A Stabilized Level. BF Decreased Correspondingly and then Grâdually Returned Towards a Stabilized Level

\subsection{Description the Structure of Model in System Identification}

In order to simplify the mathamatical analysis of the physiological system,all procedures could be described as an input-output model(black-box) between BP and BF.

An ARX model of order $(\mathrm{m}, \mathrm{n})$ and delay $d$ is deseribed by

$$
y(k)+a_{1} y(k-1)+\cdots+a_{m} y(k-m)=b_{1} u(k-d)+\cdots+b_{n} u(k-d-n+1)+e(k)
$$

where $u$ is the input, $y$ is the output and $e$ is white noise.

This is often written as

$$
A\left(z^{-1}\right) y(k)=z^{-d} B\left(z^{-} u(k)+e(k)\right.
$$

where $\mathrm{A}\left(z^{-1}\right)=1+a_{1} z^{-1}+\mathrm{L}+a_{m} \mathrm{z}^{m}, \mathrm{~B}\left(z^{-1}\right)=b_{1}+b_{2} z^{-1}+\mathrm{L}+b_{n} z^{-n+1}$ and $d$ is the shift operatior $\left(z^{-1} \mathrm{y}(k)=y(k-1)\right)$.

\subsection{Approach to Determine the Order $(m, n)$ of ARX}

Previous studies havestablished some transfer function models for analysis of blood flow autoregulation. In this paper, the order $(m, n)$ of ARX was determined by a transfer function which has been published.

Transfer unction in Figure 5 can be expressed as a quotient of polynomials:

$$
\mathrm{G}(\mathrm{S})=\frac{b_{m} s^{m}+\mathrm{L}+b_{1} s+b_{0}}{a_{n} s^{n}+\mathrm{L}+a_{1} s+a_{0}}, n \geq m
$$

Where $\mathrm{s}$ is the Laplace operator; $\mathrm{n} \geq \mathrm{m}$ according to the initial theorem and final value theorem[14].

Considering the model is an approximation of a physiology procedure,the degree of activation of the vascular smooth muscle arising through the myogenic mechanism is given by a first order transfer function[15,16]:

$$
G_{1}(s)=\frac{k_{1}}{T_{p} \mathrm{~s}+1}
$$

where $k_{1}$ is the regulation gain and $T_{p}$ is the time constant.

Based on published models on transfer function,the degree of activation arising 
through the metablic and other mechanisms why hypothesized to fit by a second-order transfer function of the form:

$$
G_{2}(s)=\frac{k_{2}}{1+2 \zeta T_{w} s+T_{w}{ }^{2} s^{2}}
$$

where $k_{2}$ is the regulation gain, $T_{w}$ is the time constant and $\zeta$ is the damping ratio.

Combined the two procedures, the autoregulation procedure of BF could be expressed by a third-order transfer function of the form[17]:

$$
\frac{B F}{\mathrm{ABP}}=G(s)=\frac{k_{p}}{\left(1+2 \zeta T_{w} s+T_{w}{ }^{2} s^{2}\right)\left(1+T_{p} s\right)}
$$

where $k_{p}=k_{1} k_{2}$

The order $(\mathrm{m}, \mathrm{n})$ of ARX can be determined by analysing the third-order transfer function.



Figure 5. Simplified Transfer Function Representation of the Relationship between BF and ABP. The Physiological System is Described As An Input-Output Model (Black-Box) Between ABR and BF. ABP Is the Input Of System And BF Is the Output

\subsection{Method of Parameter Estimation}

As for the structure of parametric identification, the task of system identification is to estimate the parameters. That is to say, we established the model of system by using input and output data to estimate the parameters. In this paper, the algorithm of parametric identification has used recursive least squares algorithm.

\subsubsection{Least Squares}

The common method in parametric estimation is using the least square method.

In order to parameter estimation, the equation (6)is written as follow:

$$
\begin{aligned}
& y(k)=-a_{1} y(k-1)-\mathrm{L}-a_{m} y(k-m)+b_{1} u(k)+\mathrm{L}+b_{n} u(k-n+1)+e(k) \\
& \varphi(k)=\left[\begin{array}{llllllll}
-y(k-1) & -y(k-2) & \cdots & -y(k-n) & u(k) & u(k-1) & \cdots & u(k-n)
\end{array}\right]^{T} \\
& \theta=\left[\begin{array}{llllllll}
a_{1} & a_{2} & \cdots & a_{m} & b_{1} & b_{2} & \cdots & b_{n}
\end{array}\right]^{T} \\
& y(k)=\varphi^{T}(k) \theta+e(k)
\end{aligned}
$$

In order to estimate parameter vector $\theta$, using all available measurements up to the current instant $k=1,2, \ldots, L$, one can first define the cost function as follow:

$$
\begin{gathered}
J=\sum_{k=1}^{L} \varepsilon^{2}(t)=\sum_{k=1}^{L}\left[y(k)-\varphi^{T}(k) \hat{\theta}\right]^{2} \\
\hat{\theta}=\Phi^{T} \Phi^{-1} \Phi^{\mathrm{T}} Y
\end{gathered}
$$

where $Y=\left(\begin{array}{llll}y(1) & y(2) & \mathrm{L} & y(L)\end{array}\right)^{T}, \Phi=\left(\varphi^{T}(1) \quad \varphi^{T}(2) \quad \mathrm{L} \quad \varphi^{T}(L)\right)^{T}$.

If this formation of least squares is used for on-line parameter estimation,we have to 
recalculate the Eq.(12) each time when a new measurement becomes available. To solve the problem of recalculating the Eq.(12), a recursive version of least squares has been proposed.

\subsubsection{Recursive Least Squares}

The basic ideal of RLS algorithm is to compute the parameter updata at time instant $k$ by adding a correction term to the previous parameter estimation once the new information becomes available. Such reformulation has reduced the computatonal requirement significantly, making the RLS extremely attractive in the last decades for on-line parameter estimation applications[18].

For the sake of easy reference, recursive expressions of a typical RLS algorithm are as follows.

$$
\begin{aligned}
K(k+1) & =\frac{P(k) \varphi(k+1)}{1+\varphi^{T}(k+1) P(t) \varphi(k+1)} \\
P(\mathrm{k}+1) & =P(k)-K(k+1) \varphi^{T}(k+1) P(k) \\
\theta(k+1) & =\theta(k)+K(k+1)\left\lfloor y(k+1)-\varphi^{T}(k+1) \theta(k)\right\rfloor
\end{aligned}
$$

\section{Results}

The result of system parameter identification is a discrete ARX model which is expressed as a quotient of polynomials. Then we use bilinean transformation method to get the continuous ARX model of the syst $m$. The pole-zere plot of the system is shown in Figure 6.

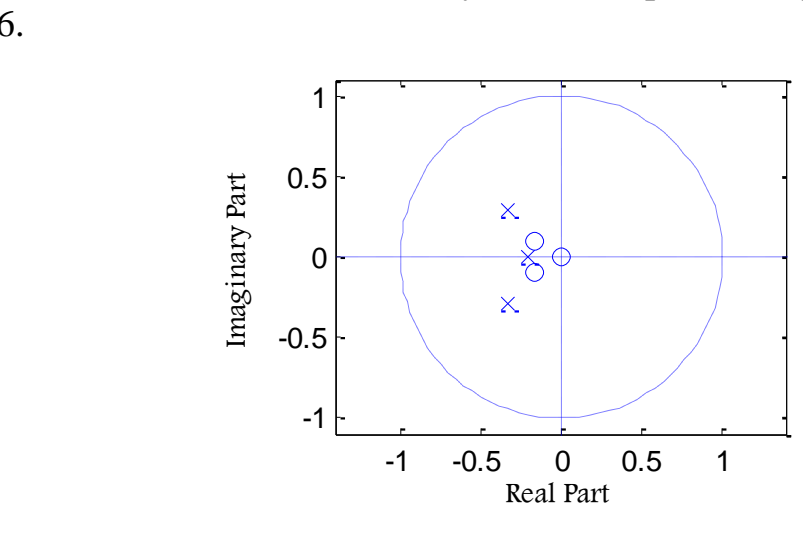

In order to judge whether this ARX model is suitable for the ocular blood flow autoregulation, in this paper the ARX model was been converted to a tranfer function.

According to the pole-zero plot of the system, the transfer function model can be witten as folloys:

$G(s)=\frac{0.1224\left(s^{2}+0.3243 s+0.0351\right)}{\left(s^{2}+0.666 s+0.1933\right)(s+0.202)}$

The equation(16) shows the parameters estimated from the experiments measured are $k p=0.1224, T p=4.9504, T w=2.2747, \zeta=0.757$.

When applying the same input to the theoretical model with these parameters, the output of the transfer function model (simulated BF) correlates with the in human experimental output (measured BF) as illustrated in Figure 7. 


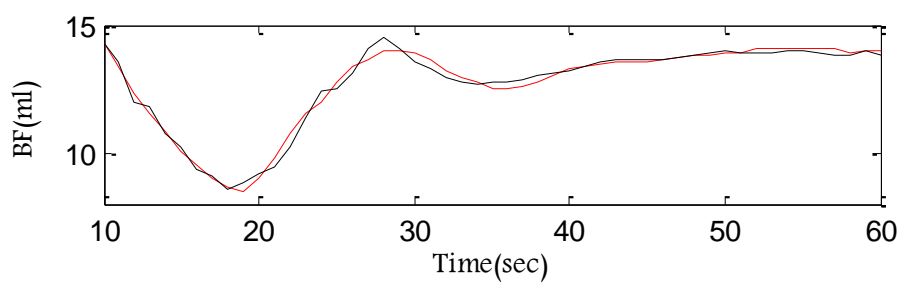

\begin{abstract}
Figure 7. The Simulated BF Response (Red Line) Fits Well With the In Humans BF Response (Black Line).As Shown In the 50 Seconds Recording (Black Curve), BF Response Induced By the BP Decrease Underwent Sar and Sar Phases. The Simulated BF Response By the Parametric Transfer Function Was Fit Well With the Experimental Derived BF Response
\end{abstract}

\title{
4. Discussion
}

In this study, an ARX model was established to describe the relationsh $\mathrm{p}$ of $\mathrm{ABP}$ and $\mathrm{BF}$ in $\mathrm{ONH}$ under the experimental condition of the thigh culf.The real parts of all poles of the denominator within Equation 4 are negative, which illustrates $\zeta \varnothing$. The system poles directly define the components in the homogeneous response. The system zeros are mostly influenced by the input of the system.

The transfer function form of ARX system model consists of 4 parameters:gain $k_{p}$, time constant $T_{w}$, time constant $T_{p}$ and a damping ratio $\zeta$. The first-order model represents the response of the vascular muscle after $\mathrm{ABP}$ (rop sharply $[19,21] . T_{p}$ is the only parameter in this model. From system simulation, it is found tha a smaller $T_{p}$ reproduces a bigger chance in BF and strongly stablizes BF to a level of baseline. The second-order model includes two parameters $T_{w}$ and $\zeta$. $\mathrm{Fom}$ the facts of dynamic response of BF, $\zeta$ means not only a damping ratio. It reflects he regulation strength of the model too.

The previous study discussed dAR and SAR including the magnitude of IOP, the response time, the recovery rate and the the magnitude of BF response[22]. There factors interaction with each pther and obsecure the interpretation of whether an autoregulation response is normal. The ARX model provided a method to detect the early or moderate change of dynamic response of certain pathologies by comparing the paremeters of the ARX model estimated from pathological condition and control condition.

The firs limitation is that the limitation of the model limited to ABP less than 80 mmHg. The model is also not fit to the situation that ABP were in the spontaneous fluctuation. The second limitation is that the model does not explain clearly the relationship between each parameter and the underlying physiological processes. The other limitation is the precision of parameters by using system identification.

In fact, the ocular BF autoregulation system is more complex than the ARX model we assumed in this paper. However, the advantage of the model couldn't be ignored. It can decribes the relationship between $\mathrm{ABP}$ and $\mathrm{BF}$ both within linear range and nonlinear ange of autoregulation.

\section{Conclusions}

The ARX model in this paper can effective describe the relationship of ABP and BF in $\mathrm{ONH}$ under the experimental condition of the thigh cuff, because it can illustrate the linear and nonlinear features of autoregulation. In this paper, we just use the linear model as the model of system identification. However, from a theoretical standpoint, ocular autoregulation is intrinsically a nonlinear phenomenon since it involves changes in vascular resistance. Future studies may also consider the extent to which time-varying parameters, or adaptive models, are required to provide a more realistic representation of 
ocular autoregulation mechanisms .

\section{Acknowledgements}

The study was supported by NIH EY019939 (LW); non restriction fund from Translational Medicine, Pfizer Inc. (LW); Good Samaritan Foundation (LW); Reserve Talents of Universities Overseas Research Program of Heilongjiang by Heilongjiang Education Department, P R China (JY).

\section{References}

[1] Y.C. Tham, X. Li, T.Y. Wong, H.A. Quigley, T.1. Aung and C.Y. Cheng, Ophthalmology, vol. 121, no. 11, (2014).

[2] A. Harris, G. Guidoboni , J.C. Arciero , A. Amireskandari , L.A. Tobe and B.A. Siesky , Eur J Ophthalmol, vol. 23, no. 2, (2013).

[3] S.S. Hayreh, Prog Retin Eye Res, vol. 20, no. 5, (2001).

[4] A.P. Cherecheanu, G. Garhofer, D. Schmidl, R. Werkmeister and L.S. Chmetterer, "Cun Opin Pharmacol", vol. 13, no. 1, (2013).

[5] . He, A.J. Vingry, J.A. Armitage and B.V. Bui, "Clinical and Experimental Optometry", vol 94, no. 2, (2011).

[6] N. Toda and M. Nakanishi-Toda, Prog Retin Eye Res., vol. 26, no. 3 (2007).

[7] B. Pemp and L. Schmetterer, Can J Ophthalmol, vol. 43, no. 3, (2008).

[8] B. Feigl, Prog Retin Eye Res, vol. 28, no. 1, 2009).

[9] J. Kur, E.A. Newman and T. Chan-Ling, Prog Retin Eye Res., vol. 31, no. 5, (2012).

[10] D. Schmidl, G. Garhofer and L. Schmetterer, Exp Eye Res., vol. 93, no. 2, (2011).

[11] A.P. Cherecheanu, G. Garhofer, D. Schmidl, R. Werkmeister and L Schmetterer, Curr Opin Pharmacol., vol. 13, no. 1, (2013).

[12] L. Quaranta, G. Manni, F. Donato and M.G. Bucci, Surv Ophthalmol., vol. 38, no.5, (1994).

[13] A. Boltz, R. Told ,N.K. Japora ,S. Palkovits R.M. Werkmer ter) D. Schmidl ,A. Popa-Cherecheanu, G. Garhöfer and L. Schmetterer, PloS one, rol.8, no. 12, (2013).

[14] Franklin, F. Gene and J. Da Abbas, "Feedback Control of Dynamic Systems", Prentice Hall, New Jersey, (2002).

[15] M. Banaji, 1. Tachtsidis, D. Delpy and S. Baigent Math Biosci., vol. 194, no. 2, (2005).

[16] N.H. Holstein-Rathlou and D.J. Marsh, Bull Math Biol., vol. 56, no. 3, (1994).

[17] J. Yu, Y. Liang, S. Thompson, G. Cull andL. Wang, Int J Physiol Pathophysiol Pharmacol, vol. 13, no. 6, (2014)

[18] J. Jiang and Y. Zhang, Computers and Electrical Engineering, vol. 30, no. 5, (2004).

[19] S.G. Diamond, K.L.Perdue and D.A. Boas, Math Biosci., vol. 220, no. 2, (2009).

[20] S.J. Payne and L. Tarassenko, Ann Biomed Eng., vol. 34, no. 5, (2006).

[21] N. Kleinstreuer, T. David, MJ, Plank and Z. Endre, "Am J Physiol Renal Physiol." vol. 294, no. 6, (2008).

[22] Y. Liang, J.C. Downs, B. Fortune, G.A. Cull, G.A. Cioffi and L. Wang, Invest Ophthalmol Vis Sci., vol. 50 , no. 5, (2009).

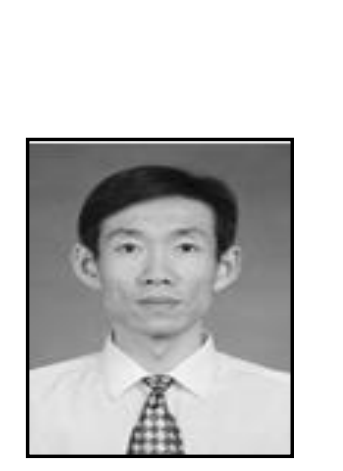

\section{Authors}

Yu Jintao, he is a Doctor, associate professor, Director of electronic and information engineering. Main research fields include signal processing of sensor, fault detection, wireless sensor networks etc. 